Peter Schaar

\title{
Datenschutz muss mit einer Stimme sprechen!
}

Unternehmen, die ihren Sitz in Schleswig-Holstein haben, müssen mit Bußgeldern bis zu $50.000 €$ rechnen, wenn sie auf ihrer Website einen "Like“-Button von Facebook anbringen. ${ }^{1}$ Dagegen raten andere Datenschutzaufsichtsbehörden den ihrer Aufsicht unterliegenden Stellen bisher nicht einmal davon ab, den "Like“Button zu verwenden.

20 Datenschutzbehörden sorgen in Deutschland dafür, dass der Datenschutz gewährleistet wird. Nun ist diese zahlenmäßige Stärke nicht gleichbedeutend mit Durchschlagskraft, denn die Datenschutzbehörden interpretieren die gesetzlichen Vorgaben bisweilen unterschiedlich. Das ist in einem föderalen Staat eigentlich nichts Ungewöhnliches und das gibt es auch in anderen Rechtsbereichen. Problematisch sind solche Meinungsunterschiede aber dort, wo die Informationstechnik überregional oder sogar global eingesetzt wird. Wenn es z.B. um die Frage geht, welche Daten in einer "Cloud“ verarbeitet werden oder wie Internetdienste zu gestalten sind. Nicht nur internationale Konzerne irritiert es, wenn mit unterschiedlichem Maß gemessen wird. Es ist der Öffentlichkeit zudem schwer vermittelbar, dass etwa der Standort eines Mar-

1 ULD an Webseitenbetreiber: „Facebook-Reichweitenanalyse abschalten“, DuD 2011 (10), S. 742 ketingbüros darüber entscheidet, ob die hamburgische oder die baden-württembergische Behörde für den Datenschutz eines global agierenden Internetkonzerns zuständig ist und mit diesem mehr oder minder tragfähige Lösungen aushandelt.

Nicht hinnehmbar ist es auch, wenn eine Landesbehörde der Kritik des zuständigen Datenschutzbeauftragten an einer Software mit dem Argument begegnet, diese sei von einem anderen Beauftragten mit einem Gütesiegel versehen worden.

Auch aus europäischer Perspektive ist diese Uneinheitlichkeit der Datenschutzpraxis mehr als misslich, zumal in Europa die Datenschutzbehörden der 27 Mitgliedstaaten unter einen Hut gebracht werden sollen. So wird darüber nachgedacht, die Art. 29 Gruppe mit einer für die Datenschutzbehörden der EU-Mitgliedstaaten verbindlichen Entscheidungskompetenz auszustatten. Es sollte auch vor diesem Hintergrund ein vitales Interesse des deutschen Datenschutzes sein, mit einer Stimme zu sprechen und auch zu handeln.

Leider haben sich die bisherigen Koordinierungsmechanismen der deutschen Datenschutzbehörden als unzureichend erwiesen, da sie auf dem Prinzip der Einstimmigkeit beruhen und Mehrheitsmeinungen allenfalls moralische Bindungswirkung entfalten.
Ohne Zweifel: Der Föderalismus hat in der Vergangenheit den Datenschutz vorangebracht und bisweilen innovative Lösungen gefördert. Auch wirkt es auf Bundes- und Landesdatenschutzbeauftragte bisweilen anspornend, wenn sie sich mit Aktivitäten anderer Kolleginnen und Kollegen messen müssen. Diese befruchtende Rolle des Föderalismus verkehrt sich aber in ihr Gegenteil, wenn im Ergebnis kein gemeinsames Handeln stattfindet oder sogar in der Öffentlichkeit der Eindruck einer Kakophonie entsteht.

Gerade wer die völlige Zentralisierung der Datenschutzaufsicht ablehnt, darf sich der Frage nicht verweigern, wie dem beschriebenen Mangel abgeholfen und die deutsche Datenschutzaufsicht gestärkt werden kann. Eine Lösung könnte darin bestehen, die Datenschutzkontrolle jedenfalls für die länderübergreifend agierenden IT-Unternehmen und für Anbieter internationaler Internetdienste an einer Stelle zu bündeln. Auch eine gesetzliche Regelung zur verbesserten Koordination der Datenschutzaufsicht mit verbindlichen Entscheidungsstrukturen würde hier weiterhelfen.

Nur eines können wir uns nicht mehr leisten: Einfach wegzusehen.

Der Bundesbeauftragte für den Datenschutz und die Informationsfreiheit 